\title{
Atomic parity nonconservation, neutron radii, and effective field theories of nuclei
}

\author{
Tapas Sil, M. Centelles, and X. Viñas \\ Departament d'Estructura i Constituents de la Matèria, Facultat de Física, Universitat de Barcelona, Diagonal 647, \\ E-08028 Barcelona, Spain \\ J. Piekarewicz \\ Department of Physics, Florida State University, Tallahassee, Florida 32306
}

(Received 5 January 2005; published 21 April 2005)

\begin{abstract}
Accurately calibrated effective field theories are used to compute atomic parity nonconserving (APNC) observables. Although accurately calibrated, these effective field theories predict a large spread in the neutron skin of heavy nuclei. Whereas the neutron skin is strongly correlated to numerous physical observables, in this contribution we focus on its impact on new physics through APNC observables. The addition of an isoscalarisovector coupling constant to the effective Lagrangian generates a wide range of values for the neutron skin of heavy nuclei without compromising the success of the model in reproducing well-constrained nuclear observables. Earlier studies have suggested that the use of isotopic ratios of APNC observables may eliminate their sensitivity to atomic structure. This leaves nuclear structure uncertainties as the main impediment for identifying physics beyond the standard model. We establish that uncertainties in the neutron skin of heavy nuclei are at present too large to measure isotopic ratios to better than the $0.1 \%$ accuracy required to test the standard model. However, we argue that such uncertainties will be significantly reduced by the upcoming measurement of the neutron radius in ${ }^{208} \mathrm{~Pb}$ at the Jefferson Laboratory.
\end{abstract}

DOI: 10.1103/PhysRevC.71.045502

PACS number(s): 24.80.+y, 21.10.Gv, 32.80.Ys, 11.30.Er

\section{INTRODUCTION}

The quest for discovering fundamental characteristics of atomic nuclei, such as their sizes and shapes, is as old as nuclear physics itself. Although for the most part this challenge has been met with enormous success, precise knowledge of certain fundamental nuclear-structure properties-among them the spatial distribution of neutrons-is still lacking. Although the original motivation for measuring the neutron density of atomic nuclei was deeply rooted in nuclear structure, the quest has been recently revived in response to the widespread impact that such a measurement will have over seemingly unrelated areas of physics. Even though the present study concentrates exclusively on atomic parity nonconservation (APNC) [1,2], a brief review of areas that could benefit from an accurate measurement of the neutron density is also presented.

Unquestionably, the accurate determination of the neutron density - and in particular its root-mean-square (rms) radiusremains a top priority in nuclear-structure physics. This inadequate state of affairs is in stark contrast to our present knowledge of proton densities. Indeed, the proton distribution in nuclei has already been mapped with exquisite precision, often much better than 1\% [3], owing to the availability of state-of-the-art electron-scattering facilities all over the world. A particularly illustrative example is ${ }^{208} \mathrm{~Pb}$, a nucleus whose charge radius is presently known with an extremely high accuracy $\left(r_{\mathrm{ch}}=5.5010 \pm 0.0009 \mathrm{fm}\right.$ [3] $)$ that exceeds at least by two orders of magnitude that of its neutron radius. Clearly, an accurate determination of neutron radii is a pressing issue. Further, without such knowledge for stable nuclei, the prospects of real progress in the future domain of nuclear structure, namely, exotic nuclei, may be seriously compromised.
Precision studies of the nucleon-nucleon $(N N)$ force is an area that has been directly affected by our poor knowledge of neutron radii. At the most fundamental level, quantum chromodynamics predicts a small flavor violation in the strong force owing to the different charges and masses of the up and down quarks. In turn, this flavor violation induces a small (of the order of $1 \%$ ) breaking in the isospin symmetry of the $N N$ force. However, to accurately quantify these "novel" isospin violations, it is necessary to have all "conventional" effects under control. A good example of this interplay between novel and conventional is provided by the Nolen-Schiffer (or Coulomb energy) anomaly. The anomaly consists in the residual discrepancy observed in the ground-state energies of mirror nuclei with a single nucleon added (or removed) from a closed shell (e.g., ${ }^{41} \mathrm{Sc}-{ }^{41} \mathrm{Ca}$ ). However, some of the conventional effects depend sensitively on our knowledge of neutron radii. Indeed, it has been argued that by using neutron skins-defined as the difference between the neutron and proton $\mathrm{rms}$ radii-significantly smaller than those predicted by theory (and apparently justified by experiment) the anomaly disappears [4].

Knowledge of neutron radii could also remarkably improve our understanding of the equation of state of neutron-rich matter. Although existing ground-state observables appear to constrain the symmetry energy of nuclear matter at a neutron density around $\rho_{n}=0.10 \mathrm{fm}^{-3}$ (as a reference value typical in nuclei) $[5,6]$, the density dependence of the symmetry energy is unknown. Recently, however, it has been found that the neutron skin of a heavy nucleus, such as ${ }^{208} \mathrm{~Pb}$, calculated with different nonrelativistic and relativistic mean field parametrizations displays a tight linear relationship with the slope of the equation of state of neutron matter (or, analogously, with the derivative of the symmetry energy), evaluated at 
$\rho_{n} \sim 0.10 \mathrm{fm}^{-3}$ [5-9]. In particular, models with a "stiffer" symmetry energy predict larger neutron skins. For instance, the neutron skin in ${ }^{208} \mathrm{~Pb}$ computed with relativistic models is larger by typically $0.1-0.2 \mathrm{fm}$ than the value obtained with Skyrme forces. In turn, the neutron skin of ${ }^{208} \mathrm{~Pb}$ is linearly correlated with the neutron skin of other relatively heavy nuclei such as ${ }^{132} \mathrm{Sn}$ or ${ }^{138} \mathrm{Ba}$. Thus, a measurement of the neutron radius of ${ }^{208} \mathrm{~Pb}$, or indeed of any other heavy nucleus, could fix a fundamental property of the equation of state.

Fixing the slope of the symmetry energy at $\rho=0.10 \mathrm{fm}^{-3}$ will also impact favorably on astrophysical observables, particularly on the structure and dynamics of neutron stars. Indeed, strong correlations between the neutron skin of ${ }^{208} \mathrm{~Pb}$ and various neutron-star properties have been established [10]. Among these, a model-independent relation between the neutron skin of ${ }^{208} \mathrm{~Pb}$ and the transition density from uniform neutron-rich matter (in the mantle) to a nonuniform phase (in the crust) was observed. Other neutron-star properties sensitive to the neutron skin of ${ }^{208} \mathrm{~Pb}$ include the radius of the star, its composition, and its cooling mechanism [11,12]. Ultimately, these correlations emerge as a result of the similar composition of the neutron skin of a heavy nucleus and the crust of a neutron star, namely, neutron-rich matter at similar densities.

Although these arguments are already compelling enough to justify the commission of new experiments to measure the neutron radius of ${ }^{208} \mathrm{~Pb}$ with unprecedented accuracy [13], this manuscript will focus on the crucial role that such a measurement could have on precision studies of the standard model, vis-à-vis, atomic parity nonconservation. The road to new physics beyond the standard model in low-energy tests in atoms, which stems from violations of fundamental symmetries that occur in the weak interaction, passes through the observation of deviations between the values measured in the laboratory and the predictions of the standard model. The effects are inherently small and, traditionally, the analysis of APNC experiments has been hindered by uncertainties in both atomic and nuclear-structure theory. However, a fruitful strategy has been devised to remove the sensitivity to atomic theory. This strategy consists in measuring ratios of parityviolating observables along an isotopic chain [14]. In this manner, uncertainties in the atomic theory factor out from the ratios. Based on their long chains of several naturally occurring isotopes, cesium, barium, dysprosium, and ytterbium appear as ideal candidates [1,2,15-18]. As a result, nuclear-structure uncertainties - primarily in the form of neutron radii-remain as the limiting factor in the search for physics beyond the standard model [19-22].

In an earlier study, a strong correlation between the neutron radius of ${ }^{208} \mathrm{~Pb}$ and the neutron radii of ${ }^{138} \mathrm{Ba},{ }^{158} \mathrm{Dy}$, and ${ }^{176} \mathrm{Yb}$ was established [23]. In that study the density dependence of the symmetry energy - and consequently the neutron radius of ${ }^{208} \mathrm{~Pb}$ - was modified through the addition of a new coupling constant $\left(\Lambda_{\mathrm{V}}\right)$ to the underlying Lagrangian $[10,11]$. This new coupling allows us to modify the density dependence of the symmetry energy without changing the saturation properties of symmetric nuclear matter. In a heavy nucleus $\Lambda_{\mathrm{v}}$ modifies the rms radius of the neutron density while leaving the rms radius of the proton density and the total binding energy practically unchanged $[10,11]$. However, the nuclear-structure model used lacked both deformation and pairing effects, which are important for the nuclei considered in APNC experiments. Thus, in Ref. [23] the calculations were limited to the study of a single member of each isotopic chain (the one with a closed neutron shell). This shortcoming is resolved in the present contribution. As a result, one is now able to map the neutron radii of these long isotopic chains as a function of both the neutron number $N$ and $\Lambda_{\mathrm{v}}$. There are previous studies of the isotopic dependence of the neutron radius in the relativistic mean-field (RMF) approximation [24,25] and of its dependence on $\Lambda_{\mathrm{V}}$ [23]. Yet the merit of the present work is that uncertainties in the neutron radius are studied for the first time as a function of both $N$ and $\Lambda_{\mathrm{v}}$ within a unified "best-fit" model. In addition, it has been recently pointed out that the accuracy in the measurements of APNC effects can be improved by using high- $Z$ atoms, such as francium, where the parity-violating effects are expected to be an order of magnitude larger than in cesium [26]. Thus we also include, in anticipation of future experiments, the analysis of the nuclear-structure corrections to the weak charge in francium isotopes.

The manuscript has been organized as follows. In Sec. II a short description of the nuclear model is given, with special emphasis paid to the treatment of deformation and pairing correlations as well as to the predictions of the model applied to cesium, barium, dysprosium, and ytterbium. In Sec. III a brief review of atomic parity violation is given, with the main goal of addressing uncertainties in APNC observables emerging from our poor knowledge of neutron radii; a detailed analysis of such uncertainties within our model is also presented in the same section. Finally, the conclusions are reserved for Sec. IV. We may summarize the two main conclusions of our work as follows. First, it appears that even when using the wealth of existing ground-state observables to constrain our nuclear-structure models, the uncertainties in the neutron radius of heavy nuclei-and consequently on APNC observables-may have been underestimated. Second, we have found, as others have before us, a tight correlation between the neutron radius of ${ }^{208} \mathrm{~Pb}$ and the neutron radii of barium, dysprosium, and ytterbium, which is shown here to hold also in deformed systems and in the presence of pairing. This correlation suggests that the nuclear-structure observables relevant for APNC will profit positively from the upcoming high-precision measurement of the neutron radius of ${ }^{208} \mathrm{~Pb}$ at the Jefferson Laboratory [13].

\section{THE NUCLEAR MODEL}

The mean-field treatment of effective field theories of hadrons, generally known as quantum hadrodynamics (QHD), is well established as a successful approach for describing diverse bulk and single-particle properties of finite nuclei and uniform nuclear matter [27-29]. These effective field theories are based on a Lagrangian density that contains the nucleon (as an elementary Dirac particle) together with an isoscalar-scalar $(\sigma)$ meson, an isoscalar-vector $(\omega)$ meson, an isovector-vector $(\rho)$ meson, and the photon as the relevant degrees of freedom for describing the nuclear many-body problem. In the meanfield approximation the meson fields are replaced by their 
ground-state expectation values, thereby becoming classical fields. The quantum structure of the theory is carried by the nucleon field. For systems with time-reversal symmetry, only the timelike component of the vector fields contribute. At the mean-field ("tadpole") level, charge conservation implies that only the third component (in isospin space) of the isovector $\rho$-meson field does not vanish. As a final product, one obtains the following Hamiltonian density:

$$
\begin{aligned}
\mathcal{H}(\mathbf{r})= & \sum_{\alpha} \varphi_{\alpha}^{\dagger}\left[-i \boldsymbol{\alpha} \cdot \nabla+\beta(M-\Phi)+W+\frac{1}{2} \tau_{3} B\right. \\
& \left.+\frac{1+\tau_{3}}{2} A\right] \varphi_{\alpha}+\frac{1}{2 g_{\mathrm{s}}^{2}}\left[(\nabla \Phi)^{2}+m_{\mathrm{s}}^{2} \Phi^{2}\right] \\
& +\frac{\kappa}{3 !} \Phi^{3}+\frac{\lambda}{4 !} \Phi^{4}-\frac{1}{2 g_{\mathrm{v}}^{2}}\left[(\nabla W)^{2}+m_{\mathrm{v}}^{2} W^{2}\right] \\
& -\frac{1}{2 g_{\rho}^{2}}\left[(\nabla B)^{2}+m_{\rho}^{2} B^{2}\right]-\Lambda_{\mathrm{v}} W^{2} B^{2}-\frac{1}{2}(\nabla A)^{2},
\end{aligned}
$$

where the summation runs over the occupied nucleon states $\varphi_{\alpha}(\mathbf{r})$ of positive energy and $\tau_{3}$ stands for the third component of the isospin operator. The scaled meson fields associated with the $\sigma, \omega$, and $\rho$ mesons are, respectively, $\Phi \equiv g_{\mathrm{s}} \phi_{0}(\mathbf{r}), W \equiv$ $g_{\mathrm{v}} V_{0}(\mathbf{r})$, and $B \equiv g_{\rho} b_{0}(\mathbf{r})$. Finally, $A \equiv e A_{0}(\mathbf{r})$ represents the timelike component of the photon field.

The variation of Eq. (1) with respect to the Dirac spinors yields the Dirac equation satisfied by the nucleons, whereas the variations with respect to the meson fields lead to the KleinGordon equations for the corresponding mesons [27-29]. The functional in Eq. (1) contains cubic and quartic scalar meson self-interactions (couplings $\kappa$ and $\lambda$, respectively) that are tuned to bring the value of the compression modulus of symmetric nuclear matter down to the empirical value ( $K=200-300 \mathrm{MeV}$ ) [30]. Further, through the inclusion of these terms one can accurately reproduce the systematics of finite nuclei throughout the periodic table as, for example, in the case of the celebrated RMF parameter set NL3 [31]. The additional isoscalar-isovector cross coupling $\Lambda_{\mathrm{V}}$ enables one to modify the density dependence of the symmetry energy without compromising the success of the model.

Insofar as we are interested in the properties of some relatively heavy nuclei that have been identified as possible candidates for APNC experiments, we extend the model of Ref. [23] to include nuclear deformation and pairing correlations in the present RMF study. Only quadrupole deformation $\left(\beta_{2}\right)$ is included though, as it is known that hexadecapole deformation is generally small for the nuclei under consideration [24]. A BCS framework is employed to deal with the pairing correlations. Note that because the nuclei to be studied lie relatively close to the $\beta$-stability line, the use of the BCS approximation is justified as the Fermi level is sufficiently bound and the mixing with continuum states-essential for drip-line nuclei-is not relevant here. As in most BCS calculations, the strength of the neutron-neutron and proton-proton pairing interactions is determined from the neutron and proton pairing gaps (respectively, $\Delta_{n}$ and $\Delta_{p}$ ) evaluated from the experimental odd-even mass differences, using a five-point formula [32].
The Dirac equation and the Klein-Gordon equations for the meson fields are treated using an axially deformed basis expansion method as described in detail in Refs. [33,34]. The calculations presented here have been carried out by expanding the fermionic wave functions and the bosonic fields in 12 and 20 oscillator shells, respectively. The solution of the equations in an axially deformed basis for the odd-even and odd-odd nuclei is more complicated. Indeed, in these cases time-reversal symmetry is broken and the odd particle induces polarization currents and time-odd components in the mean fields. However, the impact of these effects on deformation and binding energies is small [35] and will be neglected henceforth. In the pairing calculation of the odd nucleon we use the blocking approximation [36], thereby restoring time-reversal invariance in the intrinsic frame.

For the isotopic chains under consideration for APNC experiments, namely, Cs, Ba, Dy, and Yb, the NL3 parameter set yields ground-state properties, such as binding energies and deformations, in good agreement with the available experimental data [24]. For our calculations we adopt the NL3 parameter set and suitably modify it with the addition of the isoscalar-isovector coupling $\Lambda_{\mathrm{V}}$, prompted by the fact that the slope of the nuclear symmetry energy at (or near) the saturation density is at present unknown. Thus, we utilize the nonlinear isoscalar-isovector term $\Lambda_{\mathrm{V}}$ to change the density dependence of the symmetry energy, which in turn modifies the neutron radius of neutron-rich nuclei. This can be done without a significant change in those ground-state properties of finite nuclei that are well constrained experimentally [10]. In practice, for a given $\Lambda_{\mathrm{v}}$ value we readjust the nucleon-rho coupling constant $g_{\rho}$ so that the symmetry energy of nuclear matter at a density of $\rho=0.10 \mathrm{fm}^{-3}$ becomes $25.68 \mathrm{MeV}$ $[10,11,23]$. The choice is motivated by the fact that the symmetry energy of uniform nuclear matter at saturation density is not well constrained by the known properties of finite nuclei. Rather, it is some average between the bulk symmetry energy at saturation and the surface symmetry energy, which is constrained by the binding energy of finite nuclei $[5,6]$. For example, typical effective nuclear forces fitted to the empirical energies of nuclei predict a liquid-drop-model symmetry energy coefficient, which contains a surface correction, of 22-24 MeV for ${ }^{208} \mathrm{~Pb}$ [37], far smaller than the value of the bulk symmetry energy coefficient for that interaction.

Although it has been established that a variety of groundstate properties of spherical nuclei are insensitive to the choice of $\Lambda_{\mathrm{v}}[10,11,23]$, this is not the case for the neutron skin. The neutron skin $t=r_{n}-r_{p}$, where $r_{n}$ and $r_{p}$ are the neutron and proton rms radii $\left(r_{n(p)} \equiv\left\langle r^{2}\right\rangle_{n(p)}^{1 / 2}\right)$, is strongly sensitive to the density dependence of the symmetry energy and hence to the isoscalar-isovector coupling $\Lambda_{\mathrm{v}}$. This is because the slope (i.e., pressure) of the symmetry energy controls how far the neutron skin is pushed out relative to the symmetric core. To show that this scenario holds true even when spherical symmetry is broken and pairing correlations are important, we display in Fig. 1 the charge radius, binding energy, quadrupole deformation, and the neutron skin as a function of $\Lambda_{\mathrm{V}}$ for the representative heavy nucleus ${ }^{174} \mathrm{Yb}$. Note that all observables in Fig. 1 have been normalized to the corresponding NL3 values $\left(\Lambda_{\mathrm{v}}=0\right)$. It is seen that the charge radius, binding 


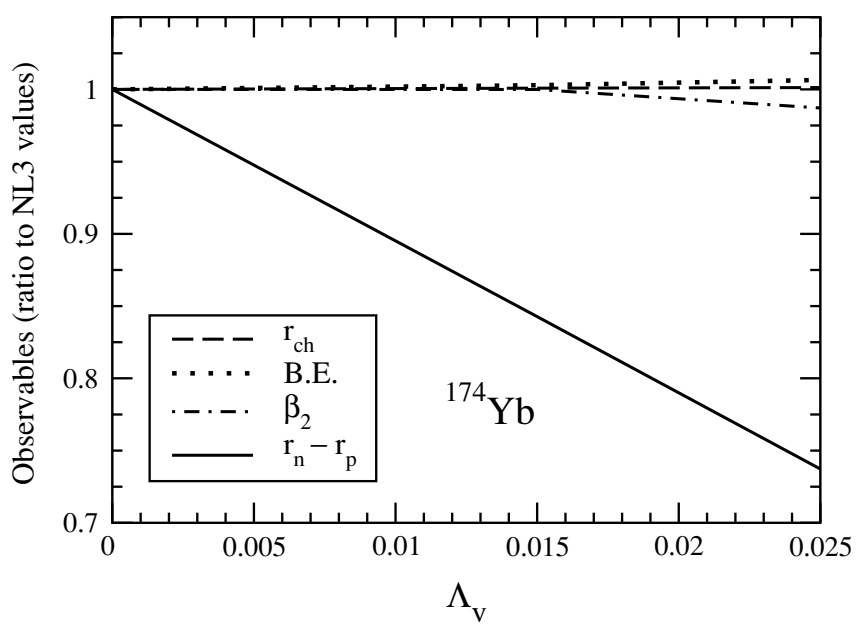

FIG. 1. The dependence of the ground-state charge radius, binding energy, quadrupole deformation, and neutron skin on the isoscalar-isovector coupling $\Lambda_{\mathrm{v}}$ is exemplified for the ${ }^{174} \mathrm{Yb}$ nucleus. The observables have been normalized to the values of the NL3 interaction $\left(\Lambda_{\mathrm{v}}=0\right)$.

energy, and quadrupole deformation are insensitive to the value of $\Lambda_{\mathrm{v}}$ in the $0-0.025$ range; the quadrupole deformation displays the largest change, but this amounts to only $2 \%$. In contrast, the neutron skin decreases rapidly with increasing $\Lambda_{\mathrm{v}}$ and it varies by about $25 \%$ when $\Lambda_{\mathrm{v}}$ is changed from 0 to 0.025 . This reflects the softening of the symmetry energy as $\Lambda_{\mathrm{v}}$ increases. Therefore, we conclude that neither pairing correlations nor deformation alter our earlier conclusions about the role of $\Lambda_{\mathrm{v}}$ in the systematics of finite nuclei.

The neutron skin of a nucleus is an important component in the study of atomic parity nonconservation. So is its charge radius. Although neutron radii of heavy nuclei $\left(r_{n}\right)$ are poorly known, high-precision data for the charge radii are available. To test the reliability of our model, we now compare in Fig. 2 calculated charge radii with experimental data for some of the relevant isotopes used in APNC experiments; these include $\mathrm{Cs}, \mathrm{Ba}$, Dy, and $\mathrm{Yb}$. The experimental values are taken from the recent compilation of Ref. [3], which combines measured data from electron scattering, from muonic x rays, and from $K_{\alpha}$ and optical isotope shifts for the purpose of providing a unified set of nuclear charge radii. Theoretical calculations are presented with the original NL3 parametrization $\left(\Lambda_{\mathrm{v}}=0\right)$ and with one for which the density dependence of the symmetry energy has been softened by fixing the value of the isoscalar-isovector coupling to $\Lambda_{\mathrm{v}}=0.025$. It can be seen that both sets of calculated charge radii are in excellent agreement with the experimental values for all of the considered nuclei. At a more quantitative level, we find that the relative $\chi^{2}$ value between the theoretical and experimental charge radii for the isotopic chains of $\mathrm{Cs}, \mathrm{Ba}, \mathrm{Dy}$, and $\mathrm{Yb}$ is always smaller than $0.25 \%$ for both $\left(\Lambda_{\mathrm{v}}=0\right.$ and $\left.\Lambda_{\mathrm{v}}=0.025\right)$ parameter sets. This result validates the use of both parameter sets in computing key nuclear observables of relevance to the APNC program. Note that the binding energies and deformations for all the nuclei considered here are also in good agreement with the experimental data (not shown). Moreover, and as alluded to earlier, these observables are highly insensitive to the choice of $\Lambda_{\mathrm{v}}$.

A more daring challenge for a theoretical model than the charge radii themselves is the reproduction of the observed charge isotope shifts. One famous case is the pronounced kink exhibited by the experimental charge radii of the $\mathrm{Pb}$ nuclei at the magic neutron number $N=126$. The kink is not reproduced by conventional nonrelativistic theories (either Skyrme or Gogny forces). In contrast, the RMF calculations show a remarkable agreement with experiment [28]. The origin for the deviations between the two models has been traced back, largely, to the isospin dependence of the spin-orbit term. We present in Fig. 3 results for the isotope shifts in two of our flagship chains for the subsequent APNC analysis: the cesium and francium alkali metals. The shifts are referred to as ${ }^{133} \mathrm{Cs}$ and ${ }^{212} \mathrm{Fr}$, respectively, as are the data of Ref. [3]. The

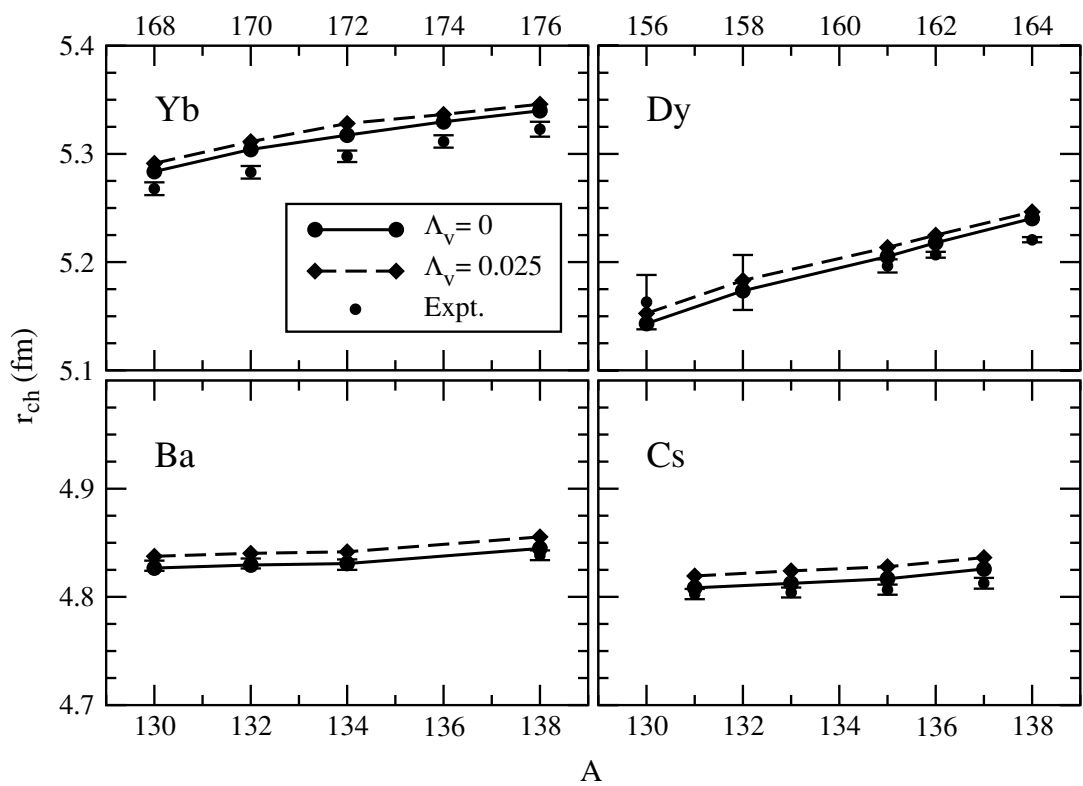

FIG. 2. Charge radii calculated with the models considered in the text are compared with the available experimental data [3] in isotope chains of interest for APNC studies. The actual experimental error bar for ${ }^{156}$ Dy and ${ }^{158}$ Dy has been reduced by a factor of 10 for display. 


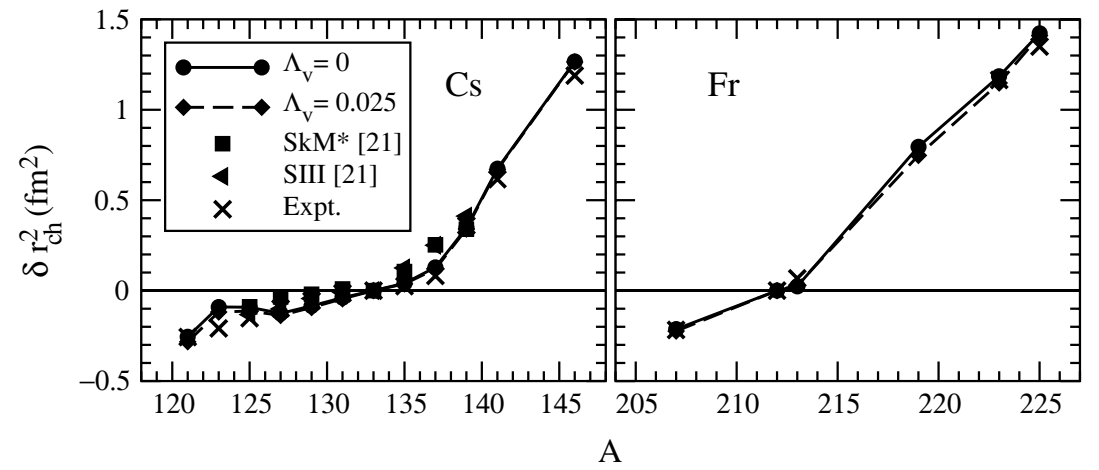

FIG. 3. Isotope shifts of the charge radii of cesium and francium nuclei. The experimental data are from Ref. [3]. experimental method of preference for the measurement of nuclear charge isotope shifts is high-resolution laser spectroscopy, which exploits the isotopic effects on the hyperfine structure of atomic optical transitions. In addition to the RMF calculations with $\Lambda_{\mathrm{v}}=0$ and $\Lambda_{\mathrm{v}}=0.025$ we have drawn in Fig. 3 the SkM* and SIII results of the nonrelativistic HartreeFock calculations of Ref. [21] for Cs. One immediately recognizes the ability of the RMF model in reproducing the trend of the changes of the experimental data when neutrons are added; conspicuously, the change of slope at the $N=82$ shell closure for $\mathrm{Cs}$ and at the $N=126$ shell closure for Fr. In spite of this, the theoretical predictions may differ from the experiment values by a factor of $\sim 2$ in some specific isotopes. One should keep in mind that this happens in cases where the isotope shift is a small quantity and that it emerges from the cancellation of two much larger numbers (the square charge radii of two isotopes). Similarly to the observables discussed here, the calculated isotope shift is a quantity that remains unaffected by the $\Lambda_{\mathrm{v}}$ coupling.

As has been mentioned in earlier publications [6-8], typical relativistic parametrizations predict a neutron skin in ${ }^{208} \mathrm{~Pb}$ of about $0.3 \mathrm{fm}$. This should be contrasted with the case of nonrelativistic forces of the Skyrme or Gogny type that yield significantly smaller values (between 0.1 and $0.2 \mathrm{fm}$ ). As the proton rms radius in ${ }^{208} \mathrm{~Pb}$ is reproduced by both relativistic and nonrelativistic models, the spread in the neutron rms radius in ${ }^{208} \mathrm{~Pb}$ - owing to the different density dependence of the symmetry energy in different theoretical models-is about $0.1-0.2 \mathrm{fm}$. Within the RMF models a spread (i.e., a theoretical uncertainty) of the neutron skin for a specific nucleus can be simulated in a controlled manner through the addition of the isoscalar-isovector coupling $\Lambda_{\mathrm{V}}[10,23]$. Starting from the original NL3 parameter set and changing the isoscalar-isovector coupling constant from $\Lambda_{\mathrm{v}}=0$ (pure NL3 interaction) to $\Lambda_{\mathrm{v}}=0.025$, the neutron skin in ${ }^{208} \mathrm{~Pb}$ varies from 0.280 to $0.209 \mathrm{fm}$ [10]. Hence, in this formalism the induced theoretical uncertainty of the neutron radius of ${ }^{208} \mathrm{~Pb}$ between these two extreme values of $\Lambda_{\mathrm{V}}$ is approximately $0.07 \mathrm{fm}$. This spread for ${ }^{208} \mathrm{~Pb}$ is only slightly larger than the expected experimental error in the measurement of the neutron radius in ${ }^{208} \mathrm{~Pb}$ via the parity-violating electron-scattering experiment at the Jefferson Laboratory (known as the PREX experiment) [13]. Thus we take the theoretical spread in the neutron radius of ${ }^{208} \mathrm{~Pb}$ as a baseline to estimate a realistic uncertainty in those nuclei involved in APNC experiments.
The neutron skins calculated with both of the relativistic parametrizations (namely, $\Lambda_{\mathrm{v}}=0$ and $\Lambda_{\mathrm{v}}=0.025$ ) for stable and long-lived isotopes of $\mathrm{Cs}, \mathrm{Ba}, \mathrm{Dy}$, and $\mathrm{Yb}$ are displayed in Fig. 4. For a specific isotope, the shift between the two curves displayed in the figure gives the model spread (due to $\Lambda_{\mathrm{V}}$ ) of the neutron skin of the given nucleus. For all the considered isotopic chains the neutron skins calculated with $\Lambda_{\mathrm{v}}=0$ (the stiffest symmetry energy) and $\Lambda_{\mathrm{v}}=0.025$ (the softest symmetry energy) lie roughly along two parallel lines. In general, the effect of $\Lambda_{\mathrm{V}}$ on the neutron skin is more prominent for the more asymmetric and heavier nuclei. This can be observed for the relatively long chain of $\mathrm{Cs}$ isotopes in Fig. 4, where the spread of the neutron skin varies approximately from 0.03 to $0.06 \mathrm{fm}$ in passing from ${ }^{123} \mathrm{Cs}$ to ${ }^{137} \mathrm{Cs}$.

To the extent that in the RMF model used in this work the proton rms radius of a given nucleus is almost independent of $\Lambda_{\mathrm{v}}$, the theoretical spread of its neutron skin is therefore approximately equal to the one for its neutron rms radius. At present, the situation regarding our ignorance of the neutron skin of heavy nuclei is unlikely to improve because the existing experimental data cannot reduce the spread in the neutron radii of $\mathrm{Cs}, \mathrm{Ba}, \mathrm{Dy}$, or $\mathrm{Yb}$ any further. Thus, we now examine whether the PREX experiment on the neutron radius of ${ }^{208} \mathrm{~Pb}$ may place important constraints on the neutron skin of APNC nuclei, including those deformed isotopes with an odd number of nucleons. Figure 5 evidences the strong linear correlation $[5,7,23]$ between the neutron skin of ${ }^{133} \mathrm{Cs}$, ${ }^{138} \mathrm{Ba},{ }^{164} \mathrm{Dy}$, and ${ }^{174} \mathrm{Yb}$ and that of ${ }^{208} \mathrm{~Pb}$. The calculations include values for the isoscalar-isovector coupling constant $\Lambda_{\mathrm{v}}$ ranging from 0 to 0.025 . Note that the correlation holds even for the case of deformed nuclei having an odd number of nucleons. With the culmination of the PREX experiment, the present theoretical spread in the neutron radius of ${ }^{208} \mathrm{~Pb}$ of $\sim 0.3 \mathrm{fm}$ (relativistic and non-relativistic models altogether) $[6,20,23]$ will be replaced by a genuine experimental error five times smaller; that is, $\Delta r_{n}\left({ }^{208} \mathrm{~Pb}\right) \approx 0.056 \mathrm{fm}$ [23]. This experimental error in the neutron radius of ${ }^{208} \mathrm{~Pb}$ will also be the experimental error in the neutron skin, as the proton radius is known with a much higher accuracy. If the expected experimental accuracy is attained, then the spread in the neutron skin of the several APNC isotopes will also be (indirectly) reduced. In turn, this result will impact favorably on APNC observables by appreciably reducing the nuclear structure uncertainty. 


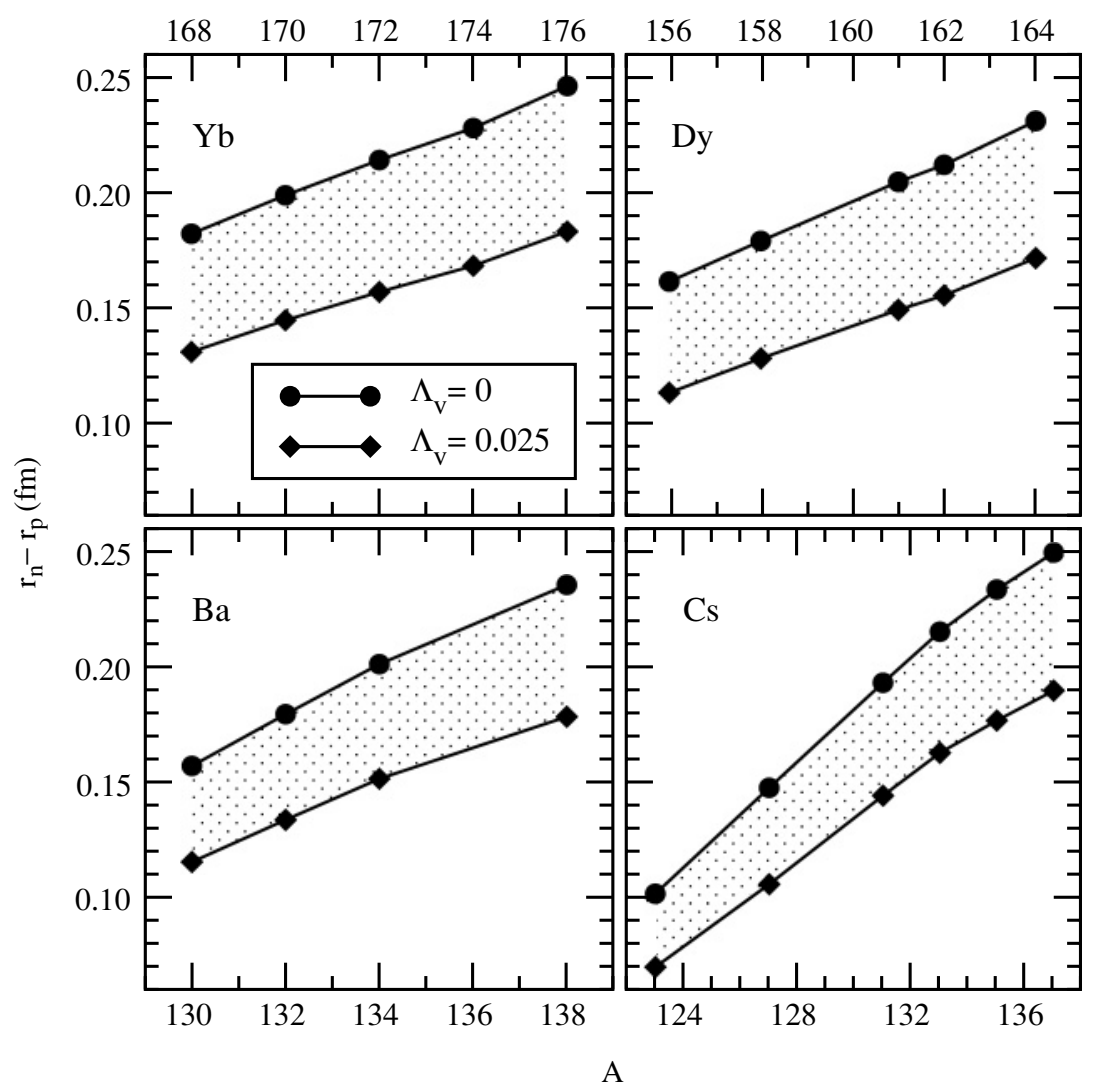

FIG. 4. Variation of the neutron skin in four isotope chains of possible relevance to APNC isotope ratios measurements for the two effective field theory models considered in this work. Note that the change in the neutron skin of ${ }^{208} \mathrm{~Pb}$ predicted by these two models, otherwise accurately calibrated for binding energies and charge radii, mocks up the uncertainty that will be left after the projected precision measurement of the neutron radius of ${ }^{208} \mathrm{~Pb}$ at the Jefferson Laboratory [13].

\section{RESULTS FOR APNC OBSERVABLES}

Parity violation in atomic systems arises from the interference between the parity-conserving electromagnetic

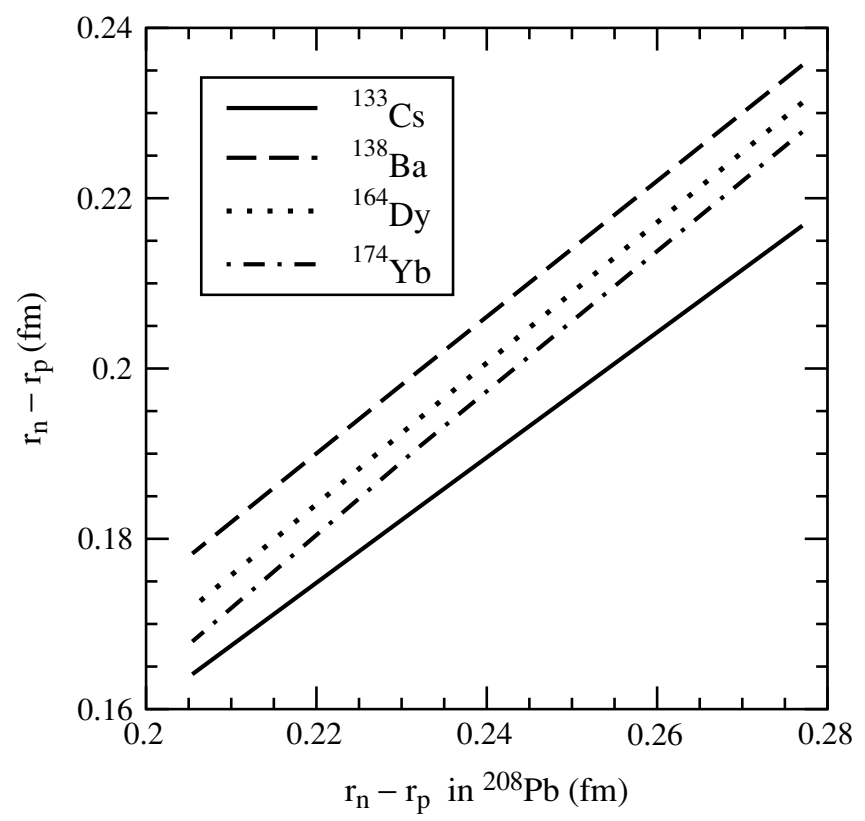

FIG. 5. Model correlation of the neutron skin of ${ }^{133} \mathrm{Cs},{ }^{138} \mathrm{Ba}$, ${ }^{164} \mathrm{Dy}$, and ${ }^{174} \mathrm{Yb}$ to the neutron skin of ${ }^{208} \mathrm{~Pb}$. The isoscalar-isovector coupling varies between $\Lambda_{\mathrm{v}}=0$ (right side of the figure) and $\Lambda_{\mathrm{v}}=$ 0.025 (left side). interaction and the parity-violating weak interaction. Although most of the binding energy of the atomic electrons comes from their attractive Coulomb interaction with the $Z$ protons in the nucleus, the weak charge of the nucleus $Q_{W}$ induces a small correction in the binding energy and parity of the electronic wave functions. These small corrections can be measured experimentally. The experimentally measured quantity is the electric dipole amplitude between two electronic states; in the absence of parity-violating effects these states would have the same parity, thereby making the amplitude vanish. The observable related to the dipole amplitude can be parametrized as follows [20-22,25,38]:

$$
\begin{aligned}
A_{\mathrm{PNC}}(N, Z) & =\xi(Z) Q_{W}(N, Z) \\
& \equiv \xi(Z)\left[Q_{W}^{\mathrm{SM}}(N, Z)+\Delta Q_{W}^{n-p}(N, Z)\right],
\end{aligned}
$$

where $\xi(Z)$ embodies the atomic structure contribution and $Q_{W}(N, Z)$ represents the weak charge of a nucleus of neutron number $N$ and electric charge $Z$.

The experimentally measured weak charge of the nucleus (without radiative corrections [20]) $Q_{W}(N, Z)$ differs from the standard model prediction,

$$
Q_{W}^{\mathrm{SM}}(N, Z)=-N+Z\left(1-4 \sin ^{2} \theta_{W}\right),
$$

by a nuclear correction factor that arises from the difference between proton and neutron one-body densities. 
That is,

$$
\Delta Q_{W}^{n-p}(N, Z)=N\left(1-\frac{q_{n}}{q_{p}}\right)
$$

with

$$
q_{n(p)}=\int d \mathbf{r} f(r) \rho_{n(p)}(r) .
$$

Note that in Eq. (3) $\theta_{W}$ is the Weinberg (or weak mixing) angle, whereas $\rho_{n(p)}$ in Eq. (5) is the neutron (proton) density normalized to one, and $f(r)$ is an electronic form factor that describes the spatial variation of the electronic axial-vector matrix element over the size of the nucleus.

The atomic parity formalism "starts" by assuming that neutrons and protons have the same spatial distribution and then adds a nuclear-structure correction factor to quantify the differences between the actual neutron and proton densities. This is motivated by the fact that the proton density, which is needed to determine various parity-conserving observables, is very accurately known. During the course of this presentation - and as has been done elsewhere [20,21] —an additional quantity that parametrizes the nuclear-structure corrections will be extensively used. This quantity is defined as follows:

$$
Q_{W}^{\text {nucl }}(N, Z)=-N\left(q_{n}-1\right)+Z\left(1-4 \sin ^{2} \theta_{W}\right)\left(q_{p}-1\right) .
$$

Note that, in terms of this quantity, the weak charge of the nucleus may be written as

$$
\begin{aligned}
Q_{W}(N, Z) & =\left[Q_{W}^{\mathrm{SM}}(N, Z)+\Delta Q_{W}^{n-p}(N, Z)\right] \\
& =\left[-N q_{n}+Z q_{p}\left(1-4 \sin ^{2} \theta_{W}\right)\right] \frac{1}{q_{p}} \\
& =\left[Q_{W}^{\mathrm{SM}}(N, Z)+Q_{W}^{\mathrm{nucl}}(N, Z)\right] \frac{1}{q_{p}} .
\end{aligned}
$$

To compute the axial form factor $f(r)$ one expands the electronic Dirac wave functions in a power series about the origin [20], assuming the Coulomb potential to be that of a uniform nuclear charge distribution. Using this prescription yields the electronic form factor in closed form:

$$
f(r)=1-\frac{(Z \alpha)^{2}}{2}\left[\left(\frac{r}{R_{p}}\right)^{2}-\frac{1}{5}\left(\frac{r}{R_{p}}\right)^{4}+\frac{1}{75}\left(\frac{r}{R_{p}}\right)^{6}\right],
$$

where $R_{p}$ is the cutoff radius of a sharp nuclear charge density.
Effects from the finite nuclear size are computed from the ground-state expectation value of the timelike component of the weak vector current by considering the neutron and proton as pointlike particles. Again, we assume sharp proton and neutron densities with rms radii equal to those predicted by self-consistent RMF models. Note that the relation between the sharp cutoff radii $R_{n(p)}$ and the corresponding rms radii $r_{n(p)}$ is given by

$$
R_{n(p)}=\sqrt{\frac{5}{3}} r_{n(p)}
$$

Estimates for the weak matrix elements $q_{p}$ and $q_{n}$ are then given by the following expressions:

$$
\begin{aligned}
q_{p} & =1-\frac{817}{3150}(Z \alpha)^{2}, \\
q_{n} & =1-(Z \alpha)^{2}\left[\frac{3}{10}\left(\frac{R_{n}}{R_{p}}\right)^{2}-\frac{3}{70}\left(\frac{R_{n}}{R_{p}}\right)^{4}+\frac{1}{450}\left(\frac{R_{n}}{R_{p}}\right)^{6}\right] \\
& =1-(Z \alpha)^{2}\left[\frac{817}{3150}+\frac{232}{525} \frac{t}{r_{p}}+\mathcal{O}\left(\left(t / r_{p}\right)^{2}\right)\right] .
\end{aligned}
$$

Although in the present approximation $q_{p}$ does not depend on nuclear-structure effects, the sensitivity of $q_{n}$ to nuclearstructure uncertainties appears in the form of the rms proton radius - which is well known - and the poorly known neutron skin $t$.

\section{A. Prospects for isotope ratios analyses}

The measurement of the weak charge in APNC experiments is plagued by theoretical uncertainties in both atomic and nuclear structure. Fortunately, uncertainties in atomic structure may be eliminated, or at least considerably reduced, by studying parity violation along a chain of isotopes and taking ratios of PNC measurements [14,19-22]. According to Eq. (2), the dependence of the parity-violating amplitude $A_{\mathrm{PNC}}$ on the atomic theory contribution $\xi(Z)$ will cancel out in the ratio of two measurements performed in two different isotopes of the same element, provided $\xi(Z)$ does not change appreciably along that isotopic chain. Thus, we now address the role of the remaining-nuclear-structure-uncertainty in APNC observables along the isotopic chains of $\mathrm{Cs}, \mathrm{Ba}, \mathrm{Dy}$, and $\mathrm{Yb}$. The observables of interest are expressed in the form of the following two ratios:

$$
\begin{aligned}
\mathcal{R}_{1} & =\frac{Q_{W}\left(N^{\prime}, Z\right)-Q_{W}(N, Z)}{Q_{W}\left(N^{\prime}, Z\right)+Q_{W}(N, Z)} \\
& =\frac{Q_{W}^{\mathrm{SM}}\left(N^{\prime}, Z\right)+\Delta Q_{W}^{n-p}\left(N^{\prime}, Z\right)-Q_{W}^{\mathrm{SM}}(N, Z)-\Delta Q_{W}^{n-p}(N, Z)}{Q_{W}^{\mathrm{SM}}\left(N^{\prime}, Z\right)+\Delta Q_{W}^{n-p}\left(N^{\prime}, Z\right)+Q_{W}^{\mathrm{SM}}(N, Z)+\Delta Q_{W}^{n-p}(N, Z)} \\
& \approx \frac{Q_{W}^{\mathrm{SM}}\left(N^{\prime}, Z\right)+Q_{W}^{\text {nucl }}\left(N^{\prime}, Z\right)-Q_{W}^{\mathrm{SM}}(N, Z)-Q_{W}^{\text {nucl }}(N, Z)}{Q_{W}^{\mathrm{SM}}\left(N^{\prime}, Z\right)+Q_{W}^{\text {nucl }}\left(N^{\prime}, Z\right)+Q_{W}^{\mathrm{SM}}(N, Z)+Q_{W}^{\text {nucl }}(N, Z)}
\end{aligned}
$$


and

$$
\begin{aligned}
\mathcal{R}_{2}=\frac{Q_{W}\left(N^{\prime}, Z\right)}{Q_{W}(N, Z)} & =\frac{Q_{W}^{\mathrm{SM}}\left(N^{\prime}, Z\right)+\Delta Q_{W}^{n-p}\left(N^{\prime}, Z\right)}{Q_{W}^{\mathrm{SM}}(N, Z)+\Delta Q_{W}^{n-p}(N, Z)} \\
& \approx \frac{Q_{W}^{\mathrm{SM}}\left(N^{\prime}, Z\right)+Q_{W}^{\text {nucl }}\left(N^{\prime}, Z\right)}{Q_{W}^{\mathrm{SM}}(N, Z)+Q_{W}^{\text {nucl }}(N, Z)} .
\end{aligned}
$$

The approximate $\operatorname{sign}(\approx)$ in these two equations follows from the assumption that the overlap $q_{p}$ appearing in Eq. (7) remains constant along the whole isotopic chain. As can be realized from Tables II and III (see later sections), this is an excellent approximation.

It has been argued in Ref. [22] that corrections to standard model predictions induced by new physics or uncertainties in nuclear structure are essentially the same whether one uses $\mathcal{R}_{1}$ and $\mathcal{R}_{2}$. Thus, we focus here exclusively on $\mathcal{R}_{1}$. Using Eqs. (3) and (6) we can write the ratio $\mathcal{R}_{1}$ approximately as

$$
\begin{aligned}
\mathcal{R}_{1} \approx & \frac{Q_{W}^{\mathrm{SM}}\left(N^{\prime}, Z\right)-Q_{W}^{\mathrm{SM}}(N, Z)}{Q_{W}^{\mathrm{SM}}\left(N^{\prime}, Z\right)+Q_{W}^{\mathrm{SM}}(N, Z)} \\
& \times\left[1+\frac{N^{\prime}}{\Delta N}\left[q_{n}\left(N^{\prime}, Z\right)-q_{n}(N, Z)\right]\right],
\end{aligned}
$$

where $N^{\prime}(N)$ is the largest (smallest) neutron number and $\Delta N \equiv N^{\prime}-N$ represents the difference in neutron number between the two extremes of the isotopic chain. Further, Ref. [22] also established that a significant test of the standard model on the basis of isotope ratios requires a measurement of $\mathcal{R}_{1}$ with an accuracy better than $0.1 \%$. Otherwise, a less precise determination of isotope ratios would not be worthwhile to compete with the sensitivity to new physics of the presently known data for the cesium isotope ${ }^{133} \mathrm{Cs}$. The measurement of the electron-nucleon parity-violating effect in ${ }^{133} \mathrm{Cs}$ to $0.35 \%$ accuracy [39] remains to date as the paradigm of PNC experimental precision.

Because atomic uncertainties have been eliminated from the ratio (14), the remaining uncertainties in $\mathcal{R}_{1}$ reflect the known accuracy in the neutron and proton rms radii. Whereas proton densities have been determined from electronscattering experiments with remarkable accuracy, knowledge of the neutron densities to a comparable level of accuracy is lacking. Thus, the main nuclear-structure uncertainty in the isotopic ratio $\mathcal{R}_{1}$ comes from our poor knowledge of the neutron radii (or neutron skin) of heavy nuclei. Indeed, by inserting Eq. (11) into Eq. (14), the relative uncertainty in $\mathcal{R}_{1}$ may be approximated by

$$
\frac{\delta \mathcal{R}_{1}}{\mathcal{R}_{1}} \approx-\frac{232}{525}(Z \alpha)^{2} \frac{N^{\prime}}{\Delta N} \delta\left[\frac{t}{r_{p}}\left(N^{\prime}, Z\right)-\frac{t}{r_{p}}(N, Z)\right]
$$

In Sec. II we showed that a spread in the neutron radius of ${ }^{208} \mathrm{~Pb}$ is obtained by using parametrizations with isoscalar-isovector couplings $\Lambda_{\mathrm{v}}=0$ and $\Lambda_{\mathrm{V}}=0.025$, which is representative of the experimental error $(\sim 1 \%)$ expected from the Jefferson Laboratory experiment. Note that the quoted error in the neutron radius of ${ }^{208} \mathrm{~Pb}$ is also the error in its neutron skin as the proton radius is known with a much higher accuracy. Inasmuch as a linear correlation between the neutron skin of ${ }^{208} \mathrm{~Pb}$ and that of the APNC nuclei has been established (see Fig. 5), an estimate of the theoretical uncertainty in the weak charge can be obtained from the spread in the neutron skin of the APNC nuclei using the $\Lambda_{\mathrm{v}}=0$ and the $\Lambda_{\mathrm{v}}=0.025$ parameter sets.

Let us outline the procedure we shall follow to determine the relative error in $\mathcal{R}_{1}$ (namely, $\delta \mathcal{R}_{1} / \mathcal{R}_{1}$ ). First, we calculate the neutron skin $t=r_{n}-r_{p}$ in a certain isotopic chain using the $\Lambda_{\mathrm{v}}=0$ parametrization. Since $r_{p}$ is roughly similar for all the isotopes of the chain, the fractional neutron skin variation along the chain is estimated as

$$
(\Delta \bar{t})_{\Lambda_{\mathrm{v}}=0}=\left.\frac{t\left(N^{\prime}, Z\right)-t(N, Z)}{\left\langle r_{p}\right\rangle}\right|_{\Lambda_{\mathrm{v}}=0},
$$

where $\left(N^{\prime}, Z\right)$ refers to the heaviest member and $(N, Z)$ to the lightest member of the isotopic chain under consideration, and $\left\langle r_{p}\right\rangle$ is the average proton rms radius of the nuclei in the chain. Next, we compute the corresponding fractional neutron skin variation $(\Delta \bar{t})_{\Lambda_{\mathrm{v}}=0.025}$ for the softer $\Lambda_{\mathrm{v}}=0.025$ parameter set. Finally, the model spread [22] is obtained as the difference between the fractional neutron skin variations calculated in the two parameter sets. That is,

$$
\delta(\Delta \bar{t})=(\Delta \bar{t})_{\Lambda_{\mathrm{v}}=0}-(\Delta \bar{t})_{\Lambda_{\mathrm{v}}=0.025} .
$$

In Table I we display the relative uncertainty $\delta \mathcal{R}_{1} / \mathcal{R}_{1}$ in the ratio $\mathcal{R}_{1}$ for each one of the isotope chains $(\mathrm{Cs}, \mathrm{Ba}$, $\mathrm{Dy}$, and $\mathrm{Yb}$ ) together with the different components required to compute it. These are the largest neutron number of the isotopic chain $N^{\prime}$, the largest difference in the neutron number of the isotopic chain $\Delta N$, the average proton rms radius $\left\langle r_{p}\right\rangle$,

TABLE I. The relative uncertainty $\delta \mathcal{R}_{1} / \mathcal{R}_{1}$ in the APNC isotope ratio $\mathcal{R}_{1}$ and the various components needed to evaluate it, as described in the text. The last row denotes the model spread $\delta(\Delta \bar{t})$, Eq. (17), that would be required to achieve $\delta \mathcal{R}_{1} / \mathcal{R}_{1}=0.1 \%$.

\begin{tabular}{lcccc}
\hline \hline Observable & Cs & Ba & Dy & Yb \\
\hline$Z$ & 55 & 56 & 66 & 70 \\
$N^{\prime}$ & 82 & 82 & 98 & 106 \\
$\Delta N$ & 14 & 8 & 8 & 8 \\
$\left\langle r_{p}\right\rangle$ & 4.752 & 4.766 & 5.134 & 5.254 \\
\hline$\delta(\Delta \bar{t})$ & 0.0059 & 0.0033 & 0.0022 & 0.0022 \\
$\delta \mathcal{R}_{1} / \mathcal{R}_{1}$ & 0.0025 & 0.0025 & 0.0027 & 0.0035 \\
{$[\delta(\Delta \bar{t})]_{0.1 \%}$} & 0.0024 & 0.0013 & 0.0008 & 0.0007 \\
\hline \hline
\end{tabular}


and the model spread $\delta(\Delta \bar{t})$. The latter turns out to be a small quantity, not only in the present relativistic calculations but also in calculations with nonrelativistic nuclear energy functionals (see Ref. [22] and references quoted therein). The reason resides in the approximately linear relationship of the neutron skin along a chain of isotopes with the neutron number. Whereas the model dependence in the value of the intercept is obvious (see Fig. 4), the slope is only mildly model dependent. And it is the difference in slope that the model spread $\delta(\Delta \bar{t})$ is sensitive to.

It is seen that the estimated relative uncertainty of the APNC ratios $\mathcal{R}_{1}$ in the four isotopic chains considered is around $0.25 \%-0.35 \%$, with a slight increase with increasing atomic number. The quoted uncertainty appears somewhat larger than the $0.1 \%$ value that would be desirable to extract new physics limits beyond the present reach of the ${ }^{133} \mathrm{Cs}$ experiment. Note that the neutron skin along a given isotopic chain varies (almost) linearly with neutron number (see Fig. 4). This makes the model spread $\delta(\Delta \bar{t})$ roughly proportional to the neutron difference $\Delta N$. Because the combination $\delta(\Delta \bar{t}) / \Delta N$ is therefore nearly independent of $\Delta N$ [38], it is unlikely that the precision in $\mathcal{R}_{1}$ can be improved by enlarging the range of the neutron difference $\Delta N$ between the two extremes of the isotopic chain [see Eq. (15)].

Table I also displays the model spread $[\delta(\Delta \bar{t})]_{0.1 \%}$ that would be required to reach the sought-after relative uncertainty of $0.1 \%$ in the isotopic ratio $\mathcal{R}_{1}$. In all cases this number is smaller than the value provided by the RMF model. Recall that we have tuned the effective interaction by means of $\Lambda_{\mathrm{v}}$ to mimic the purported $1 \%$ accuracy of the PREX experiment. Hence, it appears that under the present-and future - situation, nuclear-structure uncertainties affecting the variation of the neutron distribution along an isotopic chain are too large to make $\mathcal{R}_{1}$ a useful probe for physics beyond the standard model. However, note that the differences are not dramatic: factors of 2.5 for $\mathrm{Cs}$ and $\mathrm{Ba}, 2.75$ for $\mathrm{Dy}$, and 3 for $\mathrm{Yb}$. Thus, second-generation experiments and/or novel facilities may significantly aid in this quest.

\section{B. Prospects for single-isotope analyses}

For most of the APNC nuclei-particularly Cs and Fr, with one and no stable isotopes, respectively-one cannot envisage obtaining precision data on several members of the isotopic chain from experiments at the present time. For these cases, and to provide useful hints for future experiments, accurate estimates of the uncertainty in the neutron skin are essential. Thus, we now compute the weak charge-including nuclear and one-loop electroweak radiative corrections-for some Cs isotopes using both relativistic $\left(\Lambda_{\mathrm{v}}=0\right.$ and $\left.\Lambda_{\mathrm{v}}=0.025\right)$ parametrizations. The relevant formulas to be employed are given by Eqs. (3)-(6) suitably modified by the inclusion of radiative corrections derived by Marciano and Rosner [40]; that is,

$$
\begin{aligned}
Q_{W}^{\mathrm{SM}}(N, Z)= & (0.9857 \pm 0.0004) \\
& \times[-N+Z(1-(4.012 \pm 0.010) \bar{x})],
\end{aligned}
$$

$$
\begin{aligned}
Q_{W}^{\text {nucl }}(N, Z)= & (0.9857 \pm 0.0004)\left[-N\left(q_{n}-1\right)\right. \\
& \left.+Z(1-(4.012 \pm 0.010) \bar{x})\left(q_{p}-1\right)\right],
\end{aligned}
$$

where we have adopted the shorthand notation

$$
\bar{x} \equiv \sin ^{2} \theta_{W}=0.2323 \text {. }
$$

Notice that corrections due to new physics have not been included in these expressions; these may be found in Refs. [20,22,40].

To be able to compare more precisely our results with those in the available literature, the weak matrix elements $q_{p}$ and $q_{n}$ appearing in Eq. (19) have been slightly modified from those in Eqs. (10) and (11). We now take into account the corrections due to the finite size of the proton and neutron charge distributions, which originate additive correction terms $q_{p}^{c}$ and $q_{n}^{c}$ to Eqs. (10) and (11), respectively. These terms are given by [20]

$$
q_{n(p)}^{c}=\int d \mathbf{r} \frac{1}{6}\left\langle r^{2}\right\rangle_{I, n(p)}^{W} f(r) \nabla^{2} \rho_{n(p)}^{c} / Q_{n(p)}^{W},
$$

where $\left\langle r^{2}\right\rangle_{I, n(p)}^{W}$ are the intrinsic nucleon weak rms radii, $\rho_{n(p)}^{c}$ are the density distributions of nucleon centers, and $Q_{n(p)}^{W}$ are the nucleon weak charges. Assuming uniform nucleon distributions, we can cast the corrective terms $q_{n(p)}^{c}$ due to the finite size of the nucleon as [20]

$$
\begin{aligned}
& q_{p}^{c}=-(Z \alpha)^{2} \frac{0.32}{R^{2}}\left(2.1-\frac{0.14}{2 Q_{p}^{W}}\right), \\
& q_{n}^{c}=-(Z \alpha)^{2} \frac{0.32}{R^{2}}\left(0.74-\frac{0.14}{2 Q_{n}^{W}}\right),
\end{aligned}
$$

where $R$ denotes the sharp cutoff charge radius (which now includes the finite size of the proton) and a contribution of $0.14 \mathrm{fm}^{2}$ from the strangeness radius of the nucleon is included.

The analysis of the data of a clean measurement by Wood et al. [39] of the amplitude of the parity nonconserving transition between the $6 s$ and $7 s$ states of ${ }^{133} \mathrm{Cs}$, the only naturally occurring cesium isotope, established a value of $Q_{W}^{\exp }=-72.06(28)_{\exp }(34)_{\text {theor }}$ for the weak charge of this element [15]. This experimentally extracted result differed by $2.5 \sigma$ from the standard model prediction with radiative corrections of $Q_{W}^{\mathrm{SM}}=-73.20 \pm 0.13$ [Ref. [40] and Eq. (18)]. Let us mention that $\sigma$ is evaluated by adding in quadrature the experimental and theoretical errors quoted for $Q_{W}^{\exp }$. The excitement over this apparent disagreement has faded as a consequence of recent reports that demonstrate that the deviation between the experimental results and the standard model prediction can be removed by taking into account self-energy and vertex QED radiative corrections [41,42] (see also Ref. [2] for an up-to-date review of the status of measurements and calculations of parity violation in atoms). The newly reported values of the weak charge for ${ }^{133} \mathrm{Cs}$ - which reconcile the experiment with the standard model prediction-are $Q_{W}^{\exp }=-72.71(29)_{\exp }(39)_{\text {theor }}$ [41] and $Q_{W}^{\exp }=-72.90(28)_{\exp }(35)_{\text {theor }}$ [42]. It should be noted that in the extraction of the experimental values of the weak charge $Q_{W}^{\exp }$, one assumes equal proton and neutron densities (normalized to unity), so that the overall nuclear correction 
TABLE II. A variety of quantities of relevance to APNC (as defined in the text) for four Cs isotopes $(Z=55)$, including the sole stable element ${ }^{133} \mathrm{Cs}$. The experimental value for the weak charge of this isotope is given by $Q_{W}^{\exp }=-72.71(29)_{\exp }(39)_{\text {theor }}$ [41] and $Q_{W}^{\exp }=-72.90(28)_{\exp }(35)_{\text {theor }}[42]$.

\begin{tabular}{lccccccccc}
\hline \hline$\Lambda_{\mathrm{v}}$ & $N$ & $Q_{W}^{\mathrm{SM}}$ & $Q_{W}^{\text {nucl }}$ & $q_{n}$ & $q_{p}$ & $r_{n} / r_{p}$ & $r_{p}$ & $\Delta Q_{W}^{n-p}$ & $Q_{W}$ \\
\hline 0.000 & 76 & -71.226 & 3.284 & 0.95410 & 0.95803 & 1.04103 & 4.74141 & 0.308 & -70.918 \\
& 78 & -73.197 & 3.400 & 0.95377 & 0.95803 & 1.04568 & 4.74551 & 0.342 & -72.855 \\
& 80 & -75.169 & 3.512 & 0.95350 & 0.95803 & 1.04949 & 4.74979 & 0.373 & -74.796 \\
& 82 & -77.140 & 3.622 & 0.95327 & 0.95804 & 1.05277 & 4.75903 & 0.402 & -76.738 \\
\hline 0.025 & 76 & -71.226 & 3.228 & 0.95484 & 0.95803 & 1.03062 & 4.75244 & 0.249 & -70.977 \\
& 78 & -73.197 & 3.338 & 0.95457 & 0.95804 & 1.03449 & 4.75720 & 0.278 & -72.919 \\
& 80 & -75.169 & 3.444 & 0.95436 & 0.95804 & 1.03742 & 4.76113 & 0.302 & -74.867 \\
& 82 & -77.140 & 3.549 & 0.95418 & 0.95804 & 1.04007 & 4.76972 & 0.325 & -76.815 \\
\hline \hline
\end{tabular}

(namely $q_{p}$ ) factors out. An efficient way to account for the difference between neutron and proton densities - and thus between $q_{n}$ and $q_{p}$ - has been indicated in Eq. (4). Including radiative corrections, this nuclear-structure correction factor becomes

$$
\Delta Q_{W}^{n-p}=0.9857 N\left(1-\frac{q_{n}}{q_{p}}\right)
$$

and the total weak charge of the nucleus is computed as $Q_{W}=$ $Q_{W}^{\mathrm{SM}}+\Delta Q_{W}^{n-p}$.

Results for the weak charge together with other relevant quantities, such as $q_{n(p)}$ [including the intrinsic structure corrections (22) and (23)], the proton rms radius $r_{p}$, and the ratio $r_{n} / r_{p}$ of neutron to proton rms radii are displayed in Table II for Cs isotopes. As expected, we find that $q_{p}$ is constant, model independent, and within $0.04 \%$ of the sharpradius value expressed in Eq. (10). However, both the changes and the model dependence of the neutron radii along the isotopic chain are noteworthy. Clearly, the ratio $r_{n} / r_{p}$ increases with increasing neutron number $N$. Further, for a given neutron number $N, r_{n} / r_{p}$ decreases with increasing $\Lambda_{\mathrm{v}}$, owing to the softening of the symmetry energy. Hence, $q_{n}$ decreases with increasing neutron number and with a decreasing $\Lambda_{v}$. This ultimately leads to an increase in the nuclear part of the weak charge $Q_{W}^{\text {nucl }}$ [see Eq. (19)]. In summary, whereas $q_{p}$ is largely independent of $\Lambda_{\mathrm{v}}, q_{n}$ shows a moderate increase with $\Lambda_{\mathrm{v}}$. Thus, through variations in $\Lambda_{\mathrm{v}}$ one has a margin to adjust the nuclear contribution to the weak charge. In particular, with the proposed model dependence a change of 0.062 is induced in $Q_{W}^{\text {nucl }}$ for the ${ }^{133} \mathrm{Cs}$ atom.

The values of the nuclear-structure correction factor $\Delta Q_{W}^{n-p}$ for the studied Cs isotopes, calculated from (24), have been tabulated in Table II. For the specific case of ${ }^{133} \mathrm{Cs}$, we find a nuclear-structure correction factor of $\Delta Q_{W}^{n-p}=0.342$ $(0.278)$ for the $\Lambda_{\mathrm{v}}=0\left(\Lambda_{\mathrm{v}}=0.025\right)$ parameter set. Addition of this nuclear correction term to the standard model prediction with radiative corrections $(-73.197$, third column of Table II) ends in a total weak charge

$$
Q_{W}\left({ }^{133} \mathrm{Cs}\right)= \begin{cases}-72.855, & \text { if } \Lambda_{\mathrm{v}}=0 \\ -72.919, & \text { if } \Lambda_{\mathrm{v}}=0.025\end{cases}
$$

which is in satisfactory agreement with the measured values according to recent revisions [2,41,42].

It has been discussed in the literature [24,25] that $\Delta Q_{W}^{n-p}$ calculated with RMF parametrizations is almost twice as large as the corresponding values predicted by Skyrme interactions [21]. This is a reflection of the stiffer symmetry energy of the relativistic models. For example, by using the sharp-cutoffradius approximation of Eqs. (10) and (11) one can write

$$
\Delta Q_{W}^{n-p}=0.9857 N \frac{(Z \alpha)^{2}}{q_{p}} \frac{232}{525} \frac{t}{r_{p}} .
$$

Because this nuclear correction factor is directly proportional to the neutron skin $t$, RMF parametrizations with stiffer symmetry energies - and consequently larger neutron skinsyield larger values for $\Delta Q_{W}^{n-p}$ relative to Skyrme interactions. Equation (26) may also be used to estimate the model spread in $\Delta Q_{W}^{n-p}$ for ${ }^{133} \mathrm{Cs}$. This is given by

$$
\delta\left(\Delta Q_{W}^{n-p}\right)=0.9857 N \frac{(Z \alpha)^{2}}{q_{p}} \frac{232}{525} \delta\left(\frac{t}{r_{p}}\right) \approx 0.064,
$$

where the model spread for $t / r_{p}$ has been derived from the $r_{n} / r_{p}$ values calculated with $\Lambda_{\mathrm{v}}=0$ and $\Lambda_{\mathrm{v}}=0.025$ for ${ }^{133} \mathrm{Cs}$ (listed in Table II). A straightforward estimate of the spread computed as the difference of the values of $\Delta Q_{W}^{n-p}$ between the $\Lambda_{\mathrm{v}}=0$ and the $\Lambda_{\mathrm{v}}=0.025$ parameter sets yields

$$
\delta\left(\Delta Q_{W}^{n-p}\right) \approx 0.342-0.278=0.064,
$$

which compares well with our previous value. In Ref. [43] it has been argued that for the purpose of significantly reducing the nuclear-structure uncertainties, relative to those arising from radiative corrections, the neutron radius of ${ }^{133} \mathrm{Cs}$ must be known within $2 \%$. The promise of a $1 \%$ measurement of the neutron radius of ${ }^{208} \mathrm{~Pb}$ at the Jefferson Laboratory [13], together with theoretical correlations of the type displayed in Fig. 5, will be of considerable help in reaching the desired goal.

\section{A glimpse into the future: Fr isotopes}

Another strategy proposed as a means for attempting to improve the accuracy in APNC experiments is the utilization of high- $Z$ atoms that enhance parity-violating effects owing to 


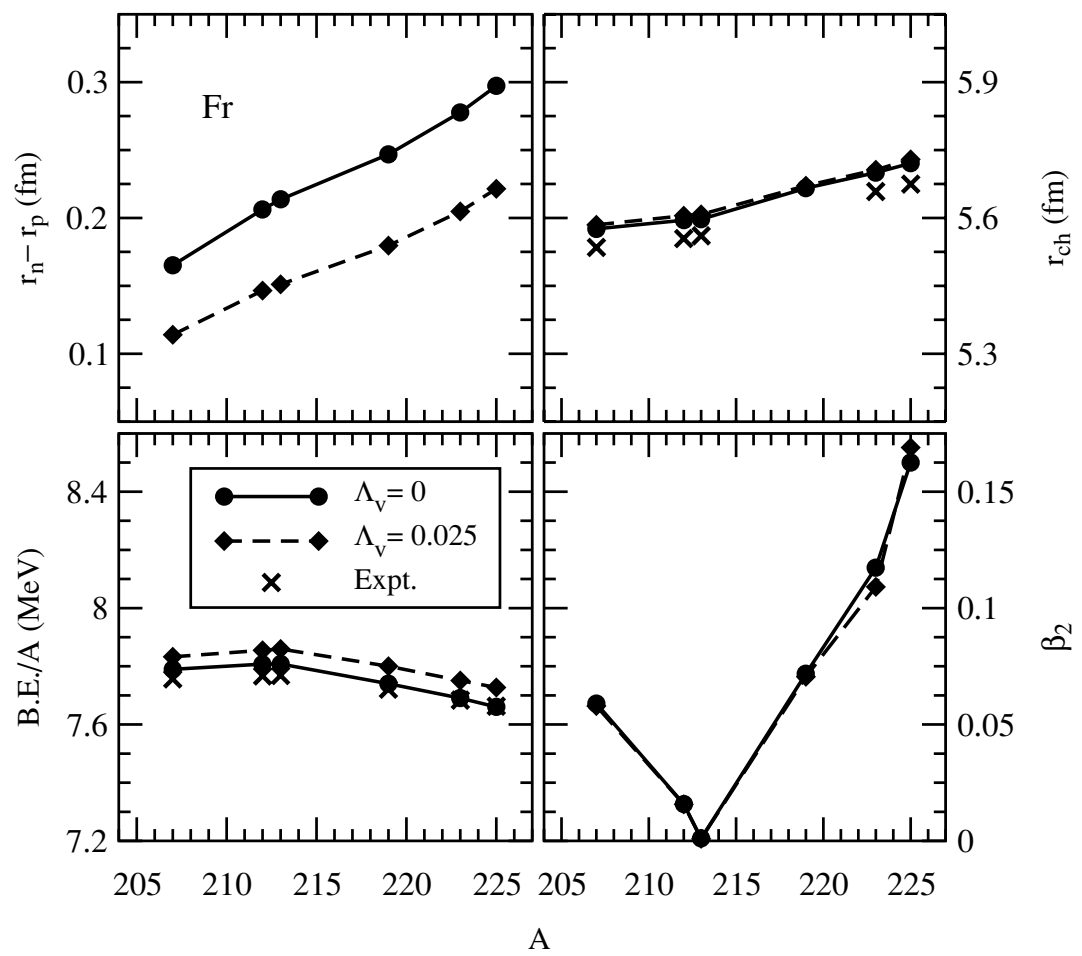

FIG. 6. Calculated ground-state properties (neutron skin, charge radius, binding energy per particle, and quadrupole deformation) of some francium isotopes. Experimental data are shown for comparison when available $[3,47]$.

both the increase in the number of nucleons and the increased electronic density in the neighborhood of the nucleus. Further, alkali atoms have the simplest atomic structure, thereby minimizing theoretical uncertainties. This makes francium, the heaviest "simple" atom, an attractive candidate for APNC experiments. Indeed, the increased number of nucleons and the increased electronic density in the region of the nucleus makes the APNC effect in Fr some 15 to 20 times larger than in Cs $[26,44]$. Unfortunately, francium has no stable naturally occurring isotopes. Although remarkable experimental progress has already been achieved in the field $[26,45,46]$, the production of intense beams of radioactive Fr isotopes represents an exciting challenge for the future of the rare-isotope facilities all over the world.

We present estimates for APNC observables in francium along an isotopic chain that ranges from ${ }^{207} \mathrm{Fr}$ to ${ }^{225} \mathrm{Fr}$ and that includes the two isotopes with the longest half-life (of about $20 \mathrm{~min}$ ); these are ${ }^{212} \mathrm{Fr}$ and ${ }^{223} \mathrm{Fr}$. In Fig. 6 we display the neutron skin, charge radii, binding energy per nucleon, and deformation for six isotopes in the chain computed with the two extremes of the values of the isoscalar-isovector coupling constant used in this work $\left(\Lambda_{\mathrm{v}}=0\right.$ and $\left.\Lambda_{\mathrm{v}}=0.025\right)$. As established earlier for ${ }^{174} \mathrm{Yb}$ in Fig. 1, the calculated values for the charge radii, binding energies, and deformation show little model dependence and reproduce the existing experimental data (where available) [3,47] rather well. As expected, it is only the (experimentally unknown) neutron skins that are sensitive to the value of $\Lambda_{\mathrm{v}}$. Note that with the exception of the semimagic nucleus ${ }^{213} \mathrm{Fr}$, the ground state of all other isotopes is found to be deformed.

As was done for the Cs isotopes in Table II, we display in Table III the calculated weak charge for the six considered Fr isotopes, alongside other relevant observables. According to Eq. (18), the standard model predictions for the weak charges of ${ }^{212} \mathrm{Fr}$ and ${ }^{223} \mathrm{Fr}$ result in $Q_{W}^{\mathrm{SM}}=-117.380 \pm 0.20$ and $-128.223 \pm 0.20$, respectively, where the uncertainty in $Q_{W}^{\mathrm{SM}}$ has been estimated in a similar way to that of ${ }^{133} \mathrm{Cs}$ in Ref. [40]. The nuclear corrections to the weak charge of ${ }^{212} \mathrm{Fr}$ $\left({ }^{223} \mathrm{Fr}\right)$ calculated from $q_{n}$ and $q_{p}$ through Eq. (24) are given as follows:

$$
\Delta Q_{W}^{n-p}= \begin{cases}1.171(1.584), & \text { if } \Lambda_{\mathrm{v}}=0, \\ 0.905(1.237), & \text { if } \Lambda_{\mathrm{v}}=0.025 .\end{cases}
$$

These results suggest that nuclear-structure uncertainties in our model, arising from differences in the shape of the neutron density relative to that of the proton, are 0.266 and 0.347 for the francium isotopes ${ }^{212} \mathrm{Fr}$ and ${ }^{223} \mathrm{Fr}$, respectively. The numbers are similar to the uncertainties 0.264 and 0.345 that one would obtain with the sharp-radius approximation of Eqs. (26) and (27).

Thus, with the expected precision to be attained by the PREX experiment, which we mimic here through a change in $\Lambda_{\mathrm{v}}$, our predictions for the weak charge $Q_{W}=Q_{W}^{\mathrm{SM}}+\Delta Q_{W}^{n-p}$ of the ${ }^{212} \mathrm{Fr}$ and ${ }^{223} \mathrm{Fr}$ isotopes vary between $Q_{W}=-116.209$ $\left(\Lambda_{\mathrm{v}}=0\right)$ and $-116.475\left(\Lambda_{\mathrm{v}}=0.025\right)$ for ${ }^{212} \mathrm{Fr}$, and between $Q_{W}=-126.639$ and -126.986 for ${ }^{223} \mathrm{Fr}$. Note that the uncertainty due to $\Lambda_{\mathrm{v}}$ should be augmented by a \pm 0.20 error from $Q_{W}^{\mathrm{SM}}$.

\section{CONCLUSIONS}

Motivated by the prospects of a high-precision (1\%) measurement of the neutron radius of ${ }^{208} \mathrm{~Pb}$ at the Thomas Jefferson Laboratory, we have examined the impact of such experiment on atomic parity nonconserving observables. Although such a measurement will have far-reaching con- 
TABLE III. A variety of quantities of relevance to APNC (as defined in the text) for six Fr isotopes $(Z=87)$. Francium has no stable isotopes.

\begin{tabular}{lccccccccc}
\hline \hline$\Lambda_{\mathrm{v}}$ & $N$ & $Q_{W}^{\mathrm{SM}}$ & $Q_{W}^{\text {nucl }}$ & $q_{n}$ & $q_{p}$ & $r_{n} / r_{p}$ & $r_{p}$ & $\Delta Q_{W}^{n-p}$ & $Q_{W}$ \\
\hline 0.000 & 120 & -112.451 & 12.650 & 0.88788 & 0.89510 & 1.02993 & 5.51834 & 0.954 & -111.497 \\
& 125 & -117.380 & 13.361 & 0.88659 & 0.89510 & 1.03724 & 5.53761 & 1.171 & -116.209 \\
& 126 & -118.366 & 13.502 & 0.88636 & 0.89510 & 1.03857 & 5.53974 & 1.213 & -117.153 \\
& 132 & -124.280 & 14.294 & 0.88544 & 0.89511 & 1.04402 & 5.60901 & 1.406 & -122.874 \\
& 136 & -128.223 & 14.866 & 0.88454 & 0.89511 & 1.04921 & 5.64364 & 1.584 & -126.639 \\
& 138 & -130.194 & 15.171 & 0.88397 & 0.89512 & 1.05246 & 5.66462 & 1.693 & -128.501 \\
\hline 0.025 & 120 & -112.451 & 12.453 & 0.88955 & 0.89510 & 1.02062 & 5.52739 & 0.734 & -111.717 \\
& 125 & -117.380 & 13.123 & 0.88853 & 0.89510 & 1.02641 & 5.54760 & 0.905 & -116.475 \\
& 126 & -118.366 & 13.250 & 0.88839 & 0.89510 & 1.02723 & 5.55034 & 0.932 & -117.434 \\
& 132 & -124.280 & 14.015 & 0.88759 & 0.89511 & 1.03199 & 5.61457 & 1.094 & -123.186 \\
& 136 & -128.223 & 14.556 & 0.88686 & 0.89511 & 1.03624 & 5.65014 & 1.237 & -126.986 \\
& 138 & -130.194 & 14.844 & 0.88637 & 0.89512 & 1.03904 & 5.67325 & 1.329 & -128.865 \\
\hline \hline
\end{tabular}

sequences in fields as diverse as nuclear structure, flavor violations in the strong interactions, and nuclear astrophysics, we have focused in this contribution exclusively on APNC observables.

Our theoretical framework is based on the highly successful relativistic NL3 parameter set, suitably modified by the addition of an isoscalar-isovector term $\left(\Lambda_{\mathrm{v}}\right)$. The virtue of such a term is that it enables one to change the neutron radius of heavy nuclei-which at present is poorly known-without compromising the success of the model in reproducing a variety of ground-state properties of nuclei throughout the periodic table. Relative to an earlier study based closely on this formalism, the present study improves on it in one essential aspect: the inclusion of deformation and pairing correlations. Without adding these effects, the predictions for the structure of most nuclei along the isotopic chains of relevance to the APNC program would be unreliable. As a result, in the present work we have mapped the neutron radii of the relevant isotopic chains as a function of both $N$ and $\Lambda_{\mathrm{v}}$. As established by others before us, we have found a strong correlation between the neutron radius of ${ }^{208} \mathrm{~Pb}$ and the neutron radius of APNC nuclei-even for the case of odd-even and odd-odd nuclei. Employing this correlation, we have used a range of values for $\Lambda_{\mathrm{v}}$ that closely matches the $1 \%$ (or $\sim 0.056 \mathrm{fm}$ ) uncertainty expected to be achieved by the Jefferson Laboratory experiment.

With this information at hand, we proceeded to study the impact of such a $1 \%$ measurement on two different combinations of APNC observables. The first set of observables $\left(\mathcal{R}_{1}\right.$ and $\left.\mathcal{R}_{2}\right)$ are formed from ratios of weak nuclear charges along isotopic chains. The merit of such observables is that the ratios are largely insensitive to uncertainties in atomic structure, leaving nuclear uncertainties-in the form of the neutron skin - as the main source of theoretical error. The second set of observables involves a direct determination of the weak charge of various alkali metals, such as cesium and francium. Unfortunately, these elements have very few stable or long-lived isotopes, so in their case the accurate determination of ratios of weak nuclear charges is more difficult.

In the case of the isotopic ratio $\mathcal{R}_{1}$, it has been claimed that a significant test of the standard model requires a determination of $\mathcal{R}_{1}$ to better than $0.1 \%$. This precision would be required for $\mathcal{R}_{1}$ to supersede the ${ }^{133} \mathrm{Cs}$ experiment as the most stringent test for new physics within the APNC program. Unfortunately, our results indicate that the projected $1 \%$ accuracy in the measurement of the neutron radius of ${ }^{208} \mathrm{~Pb}$ at the Jefferson Laboratory appears unlikely to translate into the required $0.1 \%$ (or lower) uncertainty in $\mathcal{R}_{1}$. Instead, we have established an uncertainty in $\mathcal{R}_{1}$ that is two to three times larger (of the order of $0.25 \%-0.35 \%$ ). Although the Jefferson Laboratory experiment is unlikely to achieve the desired accuracy, it is plausible that second-generation experiments may reach this goal.

In the case of the weak nuclear charge of the alkali metals, where few stable or long-lived isotopes exist, one must consider theoretical uncertainties arising from both atomic and nuclear structure. Although it is no longer possible to fully eliminate uncertainties in atomic structure (as was done for $\mathcal{R}_{1}$ ) alkali metals at least enjoy the simplest atomic structure. As far as the nuclear structure is concerned, the uncertainty is fully subsumed into a single factor: the neutron skin of the nucleus of interest. It has been argued that to reduce significantly the nuclear-structure uncertainties relative to radiative corrections, the neutron radius of ${ }^{133} \mathrm{Cs}$ must be known with an accuracy of $2 \%$ or better. Similar accuracy should be expected for the case of other alkali metals. Indeed, in anticipation of the commissioning of rare isotope accelerators all over the world and the ongoing advances in the field of production and magneto-optical trapping of radioactive atoms, we have computed the weak nuclear charge of the two longest lived francium isotopes: ${ }^{212} \mathrm{Fr}(20 \mathrm{~min})$ and ${ }^{223} \mathrm{Fr}(21.8 \mathrm{~min})$. Our assumed uncertainty in the neutron radii of ${ }^{212} \mathrm{Fr}$ and ${ }^{223} \mathrm{Fr}$ translated into a theoretical uncertainty in the value of their weak nuclear charges of $0.2 \%$ and $0.3 \%$, respectively. 
In summary, we have studied the impact of a high-precision measurement of the neutron radius of ${ }^{208} \mathrm{~Pb}$ on atomic parity nonconservation experiments. However, the relevance of such a measurement on a plethora of other research areas-such as nuclear structure, flavor violations in QCD, and neutron-star structure - has been strongly emphasized here and elsewhere. Thus, the neutron radius of ${ }^{208} \mathrm{~Pb}$ stands as one of the most fundamental nuclear physics quantities yet to be accurately determined. This unfortunate situation should be promptly corrected.

\section{ACKNOWLEDGMENTS}

This work was partially supported by Grants BFM200201868 (DGI, Spain, and FEDER), 2001SGR-00064 (DGR, Catalonia), and DE-FG05-92ER40750 (United States Department of Energy). T.S. also thanks the Spanish Education Ministry Grant SB2000-0411 for financial support. J.P. thanks the Departament d'Estructura i Constituents de la Matèria at the Universitat de Barcelona for its hospitality during the time that this project was developed.
[1] M. A. Bouchiat and C. Bouchiat, Rep. Prog. Phys. 60, 1351 (1997).

[2] J. S. M. Ginges and V. V. Flambaum, Phys. Rep. 397, 63 (2004).

[3] I. Angeli, At. Data Nucl. Data Tables 87, 185 (2004).

[4] S. Shlomo and E. Friedman, Phys. Rev. Lett. 39, 1180 (1977).

[5] B. A. Brown, Phys. Rev. Lett. 85, 5296 (2000).

[6] R. J. Furnstahl, Nucl. Phys. A706, 85 (2002).

[7] S. Typel and B. A. Brown, Phys. Rev. C 64, 027302 (2001).

[8] M. Centelles, M. Del Estal, X. Viñas, and S. K. Patra, in The Nuclear Many-Body Problem 2001, Vol. 53 of NATO Advanced Studies Institute Series B: Physics, edited by W. Nazarewicz and D. Vretenar (Kluwer, Dordrecht, 2002), p. 97.

[9] M. Baldo, C. Maieron, P. Schuck, and X. Viñas, Nucl. Phys. A736, 241 (2004).

[10] C. J. Horowitz and J. Piekarewicz, Phys. Rev. Lett. 86, 5647 (2001).

[11] C. J. Horowitz and J. Piekarewicz, Phys. Rev. C 64, 062802(R) (2001).

[12] C. J. Horowitz and J. Piekarewicz, Phys. Rev. C 66, 055803 (2002).

[13] R. Michaels, P. A. Souder, and G. M. Urciuoli, spokespersons, Jefferson Laboratory Experiment E-00-003.

[14] V. A. Dzuba, V. V. Flambaum, and I. B. Khriplovich, Z. Phys. D 1, 243 (1986).

[15] S. C. Bennett and C. E. Wieman, Phys. Rev. Lett. 82, 2484 (1999); ibid. 82, 4153 (1999); 83, 889 (1999).

[16] X. Xiong, M. He, Y. Zhao, and Z. Zhang, J. Phys. B 23, 4239 (1990).

[17] D. DeMille, Phys. Rev. Lett. 74, 4165 (1995).

[18] D. Budker, in Proceedings of WEIN 98, edited by P. Herczeg, C. M. Hoffman, and H. V. Klapdor-Kleingrothaus (World Scientific, Singapore, 1999).

[19] E. N. Fortson, Y. Pang, and L. Wilets, Phys. Rev. Lett. 65, 2857 (1990); 66, 677(E) (1991).

[20] S. J. Pollock, E. N. Fortson, and L. Wilets, Phys. Rev. C 46, 2587 (1992).

[21] B. Q. Chen and P. Vogel, Phys. Rev. C 48, 1392 (1993).

[22] M. J. Ramsey-Musolf, Phys. Rev. C 60, 015501 (1999).

[23] B. G. Todd and J. Piekarewicz, Phys. Rev. C 67, 044317 (2003).

[24] D. Vretenar, G. A. Lalazissis, and P. Ring, Phys. Rev. C 62, 045502 (2000).
[25] P. K. Panda and B. P. Das, Phys. Rev. C 62, 065501 (2000).

[26] G. D. Sprouse and L. A. Orozco, Annu. Rev. Nucl. Part. Sci. 47, 429 (1997).

[27] B. D. Serot and J. D. Walecka, Adv. Nucl. Phys. 16, 1 (1986).

[28] P. Ring, Prog. Part. Nucl. Phys. 37, 193 (1996).

[29] B. D. Serot and J. D. Walecka, Int. J. Mod. Phys. E 6, 515 (1997).

[30] J. Boguta and A. R. Bodmer, Nucl. Phys. A292, 413 (1977).

[31] G. A. Lalazissis, J. König, and P. Ring, Phys. Rev. C 55, 540 (1997).

[32] M. Bender, K. Rutz, P.-G. Reinhard, and J. A. Maruhn, Eur. Phys. J. A8, 59 (2000).

[33] P. Ring, Y. K. Gambhir, and G. A. Lalazissis, Comput. Phys. Commun. 105, 77 (1997).

[34] T. Sil, B. K. Agrawal, J. N. De, and S. K. Samaddar, Phys. Rev. C 63, 064302 (2001), and references therein.

[35] G. A. Lalazissis, D. Vretenar, and P. Ring, Nucl. Phys. A650, 133 (1999).

[36] P. Ring and P. Schuck, The Nuclear Many-Body Problem (Springer-Verlag, Berlin, 1980).

[37] M. Centelles, M. Del Estal, and X. Viñas, Nucl. Phys. A635, 193 (1998)

[38] S. J. Pollock and M. C. Welliver, Phys. Lett. B464, 177 (1999).

[39] C. S. Wood, S. C. Bennett, D. Cho, B. P. Masterson, J. L. Roberts, C. E. Tanner, and C. E. Wieman, Science 275, 1759 (1997).

[40] W. J. Marciano and J. L. Rosner, Phys. Rev. Lett. 65, 2963 (1990); ibid. 68, 898(E) (1992).

[41] M. Yu. Kuchiev and V. V. Flambaum, Phys. Rev. Lett. 89, 283002 (2002).

[42] A. I. Milstein, O. P. Sushkov, and I. S. Terekhov, Phys. Rev. Lett. 89, 283003 (2002).

[43] C. J. Horowitz, S. J. Pollock, P. A. Souder, and R. Michaels, Phys. Rev. C 63, 025501 (2001).

[44] V. A. Dzuba, V. V. Flambaum, and O. P. Sushkov, Phys. Rev. A 51, 3454 (1995).

[45] S. Sanguinetti, J. Guéna, M. Lintz, Ph. Jacquier, A. Wasan, and M. A. Bouchiat, Eur. Phys. J. D 25, 3 (2003).

[46] G. D. Sprouse, S. Aubin, E. Gomez, J. S. Grossman, L. A. Orozco, M. R. Pearson, and M. True, Eur. Phys. J. A 13, 239 (2002); S. Aubin, E. Gomez, L. A. Orozco, and G. D. Sprouse, Phys. Rev. A 70, 042504 (2004).

[47] G. Audi and A. H. Wapstra, Nucl. Phys A595, 409 (1995). 